\title{
Managing Smart Garments
}

\author{
Aaron P. Toney, Bruce H. Thomas, Wynand Marais \\ Wearable Computing Laboratory \\ School of Computer and Information Science \\ University of South Australia \\ aaron.toney@hhhh.org,bruce.thomas@unisa.edu.au,wynand.marais@gmail.com
}

\begin{abstract}
Ease of maintenance and management of smart garments (garments with integrated electronics) is crucial to their user acceptability and commercial viability. This paper presents a system that addresses user needs of easy garment charging, storage, and synchronization. The HBar smart hanger system is a novel system consisting of a set of augmented garments and coat hangers to fulfil the requirements of a smart garment management system.
\end{abstract}

\section{Introduction}

Wearable technology takes many forms, generally classifiable as either body-mounted [1] or garmentintegrated [2]. Smart garments contain garment-integrated electronics for sensing, actuation, data processing, or communication [3]. As an example, our e-Suit [4] connects to a PIM service from a PDA with a set of I/O devices integrated into the suit, LED's in the cuff, shoulder vibrotactile array display, watch display, and capacitive switches in the hem of the jacket.

Smart garments may be developed for the continuous collection of context data from the user and the user's environment. This data can be useful to other ubiquitous computing systems, but as the means of data transfer between applications increases in complexity, the user becomes less likely to facilitate this transfer on a day-today basis [5]. Commercially available smart garments are relatively rare and generally designed on the assumption that they will support a specific application or maintain a unique device. As the availability of and interest in smart garments increases, it is also reasonable to assume that users will eventually possess (and need to maintain) a larger number of garments. In order to move from ownership of a single intelligent garment to multiple intelligent garments, the means of maintaining smart garments must approximate that of conventional clothing.

Thus a successful smart wardrobe cannot treat each smart garment in the wardrobe as just another mobile device, like a phone, PDA, or camera, which the user has access to. The wardrobe as an established garment storage unit presents an opportunity to collect a large number of devices into a single management point, thus reducing the maintenance tasks required of the user. We developed the HBar (Figure 1) smart coat hanger based system to support the management and maintenance of smart garments. This system is unique in its capacity to provide both data and power simultaneously to a large number of smart garments.
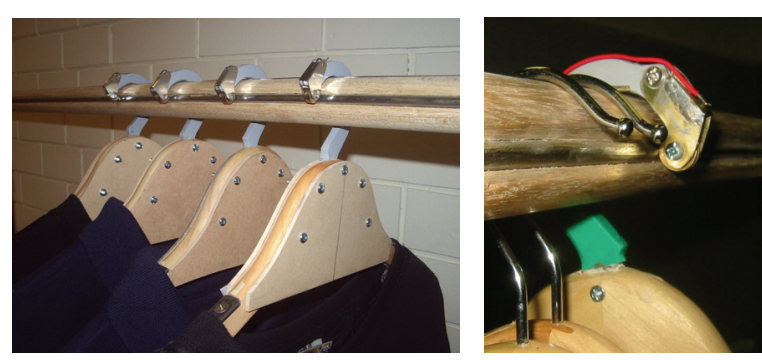

Figure 1. Picture of smart hanger

\section{Background}

One of the simplest types of smart garments are conventional garments augmented with a 'smart label'. A smart label is generally a label or patch containing a transponder, a small processor, and possibly a battery. Passive labels are parasitically powered from their environment. Active smart labels are powered by a small battery, allowing them to continuously monitor data. Currently the most common technology for a smart label is a washable RFID tag.

The inclusion of a smart label allows a smart closet or wardrobe to detect what garments it contains [6]. This functionality can be added by the garment manufacturer at a minimal cost. Automated inventory by smart label facilitates applications such as optimized home shopping [6] or suggestion of fashionable apparel.

Traditionally, garments are stored by either hanging or by folding. Purely passive devices (like passive RFID smart labels) lend themselves to either type of storage. By placing a transceiver near the garment storage area the garments can be interrogated and synchronized. However, short term storage in drawers poses a problem for active garment integrated devices requiring high charging currents, especially if there is more then one garment which requires charging. Only small amounts of power can be made available parasitically and inductive charging requires the addition of prohibitively large receiver coils in the garments [7].

Three prior projects have addressed embedding connectivity into the hanger and wardrobe space. IsoKetola et al. [7] developed a hanger-based inductive 
recharging system for smart clothing, a novel concept and prototype based on similar ideals to those presented here: a simple, easy-to-use maintenance system that builds on pre-existing concepts of garment care and storage. IsoKetola et al.'s work is primarily concerned with inductive charging and washability of smart garments; whereas our investigation is focused on conductive charging, data transmission, and maintenance of multiple smart garments. The external plug on the hanger of the IsoKetola et al.'s system restricted the scalability of the system, since a wardrobe cupboard would require a plug for each hanger.

Wan [6] describes the development of a smart-wardrobe concept. The Accenture "Magic Wardrobe" is focused on a home-shopping concept using RFID tags. The wardrobe is aware of its contents, and uses a web interface to suggest garment pairings or items of clothing for purchase. This paradigm assumes standard garments, with no intelligence or electronic capability beyond the integration of RFID tags.

Matthews et al. [8] explored the use of a collection of hangers to form an ambient display. Their system, like ours, used a one wire bus to both parasitically power and detect the hangers currently present within the wardrobe. Mathews' work restricted hanger insertions and removals so that hanger order would be known. Taken as a collection with a known ordering, these LEDs integrated into the Matthew's hangers formed pixels within a simple ambient display. As Matthews' work focused on ambient displays their system, and unlike ours, did not support the garments. Specifically their hangers were unable to detect, communicate with, or charge garments. Since their work was not required to support garments they were able to use the Maxim one-wire ${ }^{\mathrm{TM}}$ technology. This approach does not allow garments to announce themselves when plugged into the bus, and can only supply very small amounts of power for running and charging devices on the bus. The HBar system developed a custom one wire bus protocol in order to meet these needs.

\section{System Design}

The HBar system consists of a collection of smart garments and hangers which communicate over a one wire bus integrated into a closet rod. An optional bus master acts as the gateway between the devices on the bus and any software management system.

\subsection{Design requirements}

The primary objective of the HBar system is to fulfil the maintenance and management requirements of smart garments with minimal impact on the existing physical relationships between users and their clothing. This is achieved by incorporating our infrastructure into a standard garment storage system, such as clothes hangers and a wardrobe. Table 1 outlines the goals and objectives considered in designing the HBar hangers.

To effectively manage and maintain smart garments the following four core tasks are essential: Synchronization: The system must be able to synchronize all data on the garments with a central garment information system. This requires the smart garments to be individually identified, and the capacity and configured role of each garment to be discovered. Recharging: For powered smart garments the system must be able to recharge the garment when needed. Agency: The user's wardrobe must provide a front end for high level functionality. As a collection of smart garments, the wardrobe represents a single device with the potential to reduce the cognitive demands of controlling a large number of devices. Diagnostics: Whenever the garment is returned to the system after being worn or cleaned, the system must check that the smart garment is still functioning properly.

1. Individual hangers must be inexpensive.

2. Hangers must be able to slide along the closet bar to enable searching, sorting, and compacting the wardrobe.

3. Hangers must be able to be removed and replaced in any order.

4. Garments must be able to be hung on the hanger facing in either direction.

5. Hanging garments must not require extensive or precise user participation.

6. Smart hangers must be capable of operating while co-mingled with standard (non-smart) hangers.

Table 1. Expectation of Conventional Hangers

The HBar system provides its users with a single interface to their collection of smart garments. For the foreseeable future, users of smart garments will also possess standard garments. Thus smart garment management systems will be required to support both conventional garments and hangers.

The following objectives of the garment connection were identified, aiming to approximate the ease of use of standard garments: 1) Electrical connection must be made by simply placing the garment on the hanger, without the use of plugs or sockets. 2) The connection must not require extensive adjustment of the garment on the hanger. 3) The electrical connection must be reliable and consistent, even when hangers are jostled, moved, or compressed. 4) The garment connector must have minimal impact on the garment's comfort and appearance. 5) The garment connector must provide consistent contact with the hanger regardless of garment shape, type, construction, or materials.

\subsection{Electrical and mechanical connection}

Reliable electrical connection between the fabric contacts and the hanger is crucial to the operation of the 
system. The garment makes contact with the hanger through two $12 \mathrm{~cm}$ by $1.5 \mathrm{~cm}$ steel contacts on either shoulder of the hanger (Figure 2). Direct electrical contact is made between the garment and the one wire bus through patches of conductive fabric sewn into both shoulders of the garment (Figure 2 and Figure 3 ). The conductive patches were made from silver coated fabric. The coated fabric was composed of $92 \%$ Nylon and $8 \%$ Dorlastan [9] and connected to the garment electronics by conductive thread [10].

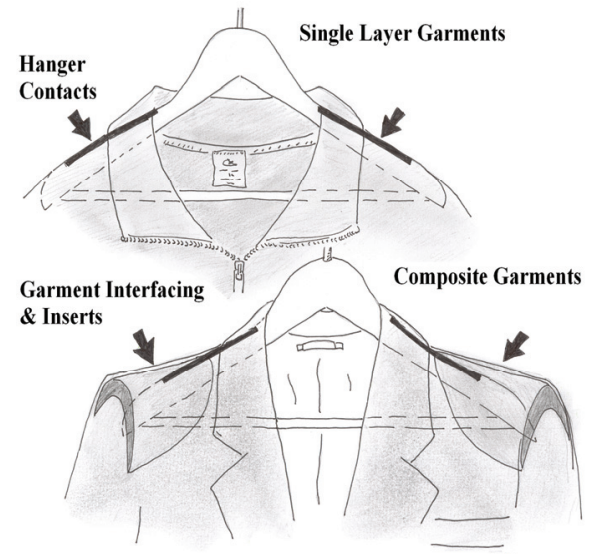

Figure 2. Garment Contacts

Fabric patches add minimal bulk, even in single-layer garments. It is crucial that contacts be sized and located such that a reliable electrical connection is formed through "normal" storage of the garment without requiring additional user interaction. Increasing contact depth will increase the probability of garment bus connectivity. Unfortunately increasing the depth of the contact is not always appropriate as it may impact the garments comfort or appearance.

The HBar hanger's hook connects to a one wire bus for data and power. The HBar hanger is designed to gravitationally align with the HBar closet bar. Once hung up, with or without a garment, the hanger settles into the desired operating position on the bar. Contact is made with the closet bar in two places; through a ground return connection and through a spring loaded contact for the data/power connection (Figure 1-Right). The ground connection rises above the top of the bar. The data/power connection is recessed and is connected via the spring loaded contact. The recessed connection maximises the isolation of the bus, and minimises the potential for shorts.

\subsection{Bus arbitration and protocol}

A device on the HBar bus uses an open collector connection to for active low bus assertion. Unlike most one-wire buses the HBar buses do not have a bus master. Bus arbitration is carrier sense multiple access with collision avoidance (CSMA/CA). During transmission the bus state is monitored and in the event that the device detects the bus being asserted during an expected deassertion time a collision is assumed. If the bus remains asserted for longer then a byte width either a bus short, a reset, or another device performing a high current charge is assumed. In such an event the HBar device enters a low power state and waits, for a period of time derived from its unique ID, before attempting retransmission. The HBar bus uses a protocol with 3 byte packet headers that can contain from 0-7 additional bytes. Each packet contains a one byte source and destination address. These address bytes are followed by five configuration and status bits and three successive bits indicating the number of accompanying data bytes in the current packet.

\subsection{Evaluation \& Lessons Learned}

Several prototypes (Figure 3) were constructed to test the smart hanger's mechanical and electrical contacts. All prototypes were built from conventional T-shirts. Conductive steel pads were added to both shoulders of the system's hangers; one pad connected to the bus' ground return line and, the other to the power and data bus. The T-shirts in turn had conductive fabric patches sewn into either shoulder in order to provide a conductive connection to the hanger's pads.

In order to evaluate reliability of alignment and electrical contact in both the hangers and the smart garments, a small user study was performed. Seven subjects were each given a smart hanger. They were asked to repeatedly hang the garment up in the system, testing one hundred and eleven garment insertions. The hangers repeatedly broadcast a packet announcing its presence on the bus once every $420 \mathrm{mS}$. Successful reception of these packets signalled an insertion. The id of the device being inserted was read from the received packet. The system successfully recognized 110 of 111 insertions. The missed insertion resulted from a mechanical failure within the hanger head spring mechanism.

There were several important lessons learned both from observing subjects in a small user study and from ongoing informal evaluation and development of the system. First: during insertion searching, sorting, and compacting of the wardrobe hangers are slid from side to side and rotated. There is a settling time after any hanger motion where bus reliability is variable. As a result insertions in our study required a varying length of time to be recognized. The longest time required to recognize an insertion, which should give an initial estimate of desired settling time for this design, was 9 seconds. Most insertions were recognised within 1 to 2 seconds.

Second: initially it was believed that the hang weight of lighter garments would be insufficient to cause reliable electrical contact between the garment and the hanger. As prototype development progressed it became clear that 
compared with the $100 \Omega-1 \mathrm{~K} \Omega$ resistance of the data bus, variations in the resistance in the hanger to garment connection was a negligible source of error. The hanging weights of the light prototype T-shirts proved sufficient to provide reliable contact between the garment's and the hanger's pads.

Third: since contacts saddle the coat hanger when hung up, the garment integrated pads should be narrow and deep straps running over the shoulder. The 'front to back' depth of the contacts was discovered to be much more important then the 'side to side' width in maintaining a reliable electrical contact with the hangers. The deeper the conductive pad the less attention users will have to pay to garment alignment. To maximize contact area future prototypes will use thin deep strips as opposed to the wide and shallow approach taken by the current prototypes.

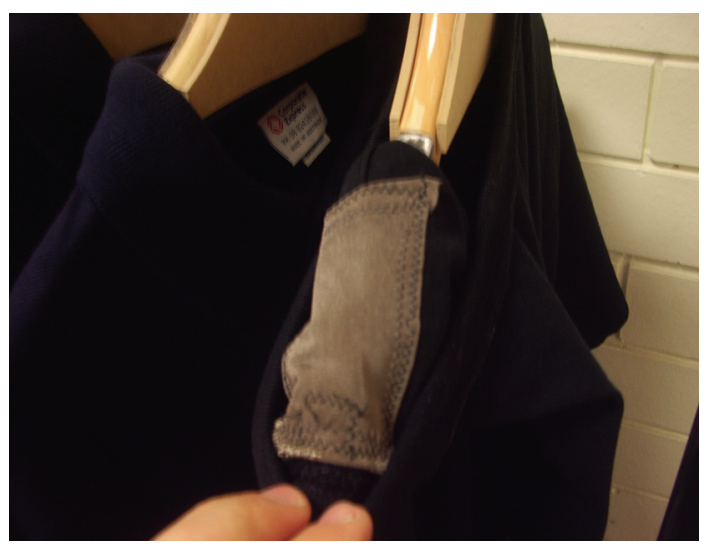

Figure 3. Garment Conductive Pads

Finally: In the future the use of computer controlled production methods will be required for construction of prototypes. Currently the hangers used were all hand built prototypes. As a result of every hanger being similar but unique the system required being recalibrated when the number of hangers being tested was increased. This became problematic as the research progressed from using a single hanger to using multiple hangers simultaneously.

\section{Conclusion}

This paper presents the HBar smart hanger system for the storage and management of smart garments. The system consists of smart hangers, a one wire bus integrated into a closet bar, and a software management system. The requirements for electrical connections in smart garments have been outlined. The HBar system demonstrates connecting both smart hangers and garments to a one wire bus for data and power. The one wire bus integrated into the closet bar provides a physical connection for smart garments to recharge and transmit data. A basic garment management system has been developed as a proof of concept demonstrating the following functionality:

1. Detects both smart garment and hangers being inserted and removed from the wardrobe.

2. Parasitically powers multiple smart devices connected to the bus.

3. Provides communication between multiple devices connected to the bus.

A custom wardrobe is in the process of being constructed to facilitate further development with more rigorous testing of the HBar system.

\section{Acknowledgements}

The authors want to acknowledge technical contributions made to this project by Lucy E. Dunne and Ross T. Smith. Additionally the authors would like to acknowledge the many excellent editorial and content suggestions made by Benjamin Avery, Peter Hutterer, Mark Rebane, and our paper submission coordinator.

\section{REFERENCES}

1. Bodine, K. and F. Gemperle. Effects of functionality on perceived comfort of wearables. in the International Symposium on Wearable Computers. 2005: IEEE.

2. Dunne, L.E., S.P. Ashdown, and E. McDonald. "Smart Systems" Wearable Integration of Intelligent Technology. in First Conference of the International Center for Excellence in Wearable Electronics and Smart Fashion Products. 2002. Cottbus, Germany.

3. E. R. Post, et al., E-broidery: Design and fabrication of textile-based computing. IBM Systems Journal, 2000. 39(3\&4): p. 840-860.

4. Toney, A.P., et al., Designing to minimize the social consequences arising from technology use by the mobile professional. Special Issue of Personal and Ubiquitous Computing, Springer-Verlag London, 2002.

5. Pentland, A. Smart Rooms, Smart Clothes. in 14th International Conference on Pattern Recognition, 1998

6. Wan, D. Magic Wardrobe: Situated Shopping from Your Own Bedroom. in HUC. 2002. Goteborg Sweden.

7. Iso-Ketola, P., et al. A Mobile Device as User Interface for Wearable Applications. in PERMID. 2005. Munich, Germany: LMU Munich.

8. Matthews, T., et al., Augmenting Collections of Everyday Objects: A Case Study of Clothes Hangers As an Information Display. Pervasive Computing. Vol. 3001. 2004, Austria: Springer. P340-344.

9. LessEMF.com, EMF Shielding \& Conductive Fabrics - http://www.lessemf.com/fabric.html.

10. Lifesaver, L., Conductive Thread for Lame Repair http://members.shaw.ca/ubik/thread/. 